\title{
Comparing Three Protocols of DODV, DSR, DSDV FOR VANET NETWORK
}

\author{
${ }^{1}$ Hossein Shahbazi and ${ }^{2}$ Sepideh Jamshidinejad \\ ${ }^{1}$ Master's student of Information Security at MehrAstan University \\ ${ }^{2}$ Ph.D. student of Computer Engineering, Islamic Azad University of Rasht
}

\begin{abstract}
An ad hoc network is a network created by wireless hosts that can be mobile. This means that no infrastructure such as a central station, router, roaming, or anything else used by other networks to help network structure or anything else that other networks use to help Network structure, is not used. Rather, they are just a few wireless nodes connecting to non-neighboring nodes with the help of the neighboring nodes. Wireless ad hoc mobile network (MANET) and Wireless ad hoc inter-car network (VANET), are among wireless networks which have the most effective parameters in the ability of this type of networksto transfer multimedia from source to destination. In this research, we examine the challenges and solutions presented in DODV, DSR, and DSDV protocols.
\end{abstract}

\section{KEYWORDS:}

Ad-hocwireless network, DODV, DSR andDSDV protocols

\section{INTRODUCTION}

Today, the use of wireless communication systems has become a common mode among the public. Therefore, most researchers focus their attention on conducting research on ad hoc networks that require no communication and management infrastructure to provide ease of use and optimize the performance of these networks. Ad hoc networks are a kind of wireless independent network, which is automatically managed by a set of mobile nodes without the use of a central infrastructure or control. The relationship between nodes in this network is somehow based on the trust and interaction between nodes [1].

VANET is a sample of adhoc Networks that uses the cars in the path to exchange information instead of using wireless systems. It should be noted that some of the most important challenges for the advancement of these networks are the discussion of the routing protocol, information security and authentication of new vehicles in the network [2].

MANET consists of a number of mobile wireless nodes that can communicate with each other without any network infrastructure or centralized management. In this type of network, each mobile node performs sending information packets not only as hosts, but also as routers. Therefore, each node must discover and maintain a path to other nodes. Due to the mobility of the nodes, the Ad Hoc network routers often need to reflect the network topology changes to the 
nodes. For this reason, the design of a routing protocol for wireless networks is much more challenging than wired networks [2].

\section{ROUTING}

The process of transferring information from one source to a given destination is called the routing of information packets in the network, which is the main task of the network layer. Creating this path depends, in the most important, on the routing algorithm used. In all networks, including fixed and mobile, routing is one of the most important issues and various protocols for this task are provided [2].

\subsection{Routing Protocols}

VANETs are a specific category of ad hoc Networks in a particular area. The main differences between VANET and MANET are the mobile pattern and rapidly changing topology. Normal ad hoc routing protocols are first tested in MANETs and then evaluated for use in the VANET environment. A routing protocol is monitored by the method of communicating between various components and objects for the exchange of desired information in a significant amount of time. In Figure 1, we show the mobile ad hoc network routing protocols.

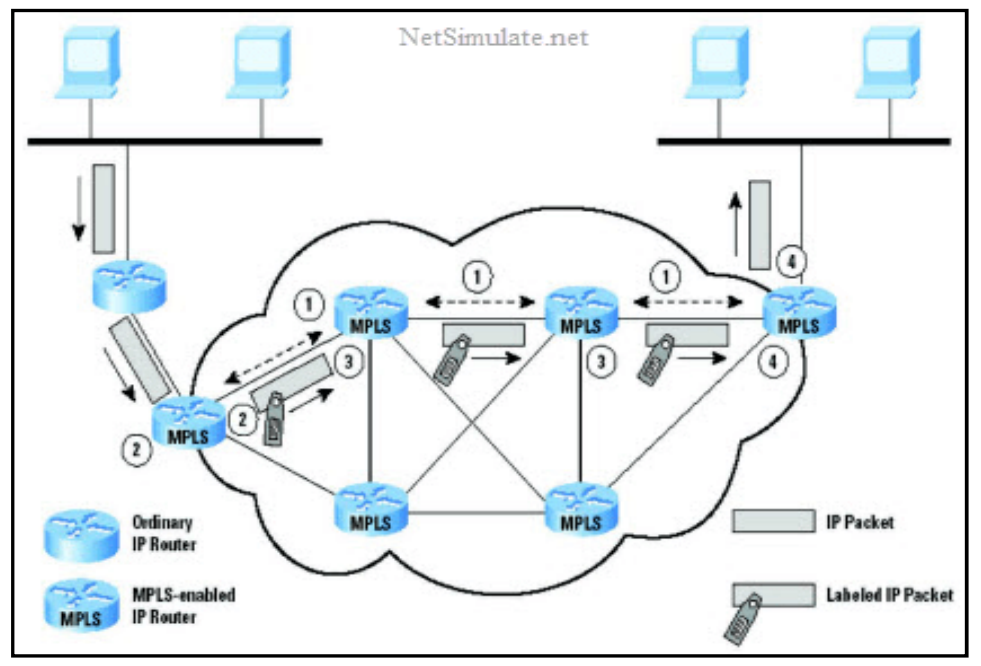

Figure 1.MANET mobile ad hoc routing network protocols[12]

Figure 2 depicts the hierarchy of VANET routing protocols that we can categorize on a general scale:

- Location-based routing protocol (geographic).

- Topology-based routing protocol.

- Broadcast-based routing protocol.

- Clustering-based (cluster) routing protocol.

- Geographical distribution-based routing protocol 


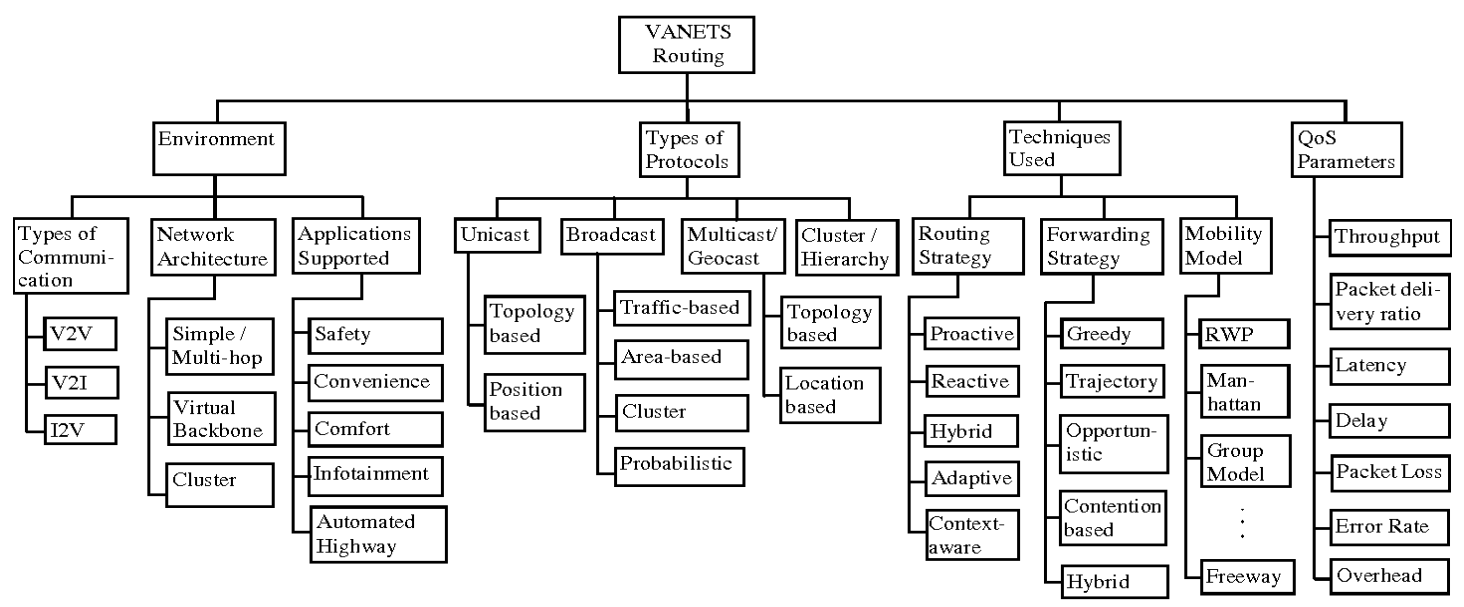

Figure2. Taxonomy of routing in VANETs [3]

In this research, we will only consider DSDV, AODV, and DSR topology routing protocols [2].

\subsubsection{DSDV routing protocol}

The DSDV, or Destination Sequence Distance Vector, is referred to as the Destination Sequence Distance, and is a real-time routing protocol in which each node maintains a table of information in the presence of any other node in the network. This protocol updates the table periodically whenever a change is made to the network. If any changes occur in the network, it will be communicated to all nodes in the network. The distance vector algorithm is based on the destination order number, a step-by-step routing protocol that uses the extended from Bellman Ford routing mechanism. In this way, each node broadcasts the path updates and guarantees the freedom of the circles. Each node maintains a routing table that includes destinations, the number of hops to reach each destination, and the order number corresponding to the destination node. This sequence number is used to specify the old paths. In fact, any route whose destination number is larger is a new direction, and this is used to avoid creating a loop, so that always routes that have a larger order number are used. The reason that the use of old paths may create loops is because nodes in the ad hoc network are constantly changing, a node may be displaced so that it can be placed in the path of the loop. Route recovery is sent over the entire network [2].

\subsubsection{AODV routing protocol}

AODV or Ad-hoc On Demand Distance is referred to as the single-useddistance vector on demand, and is a passive routing protocol that establishes a path to a destination when the demand for data transfer occurs. This protocol does not have a loop. The AODV routing protocol uses the constant number of message pairs $<$ RREQ, RREP $>$ to find the path. AODV updates only the related adjacent nodes instead of informing all the nodes in the network. The basis of this protocol is based on public key cryptography. This protocol is established to provide security in AODV algorithm as well as the ARAN protocol. AODV routing messages are path request messages, path responses, and path errors that use digital signatures to assure the integrity and authenticity of their credentials [2]. 


\subsubsection{DSR routing protocol}

DSR is referred to as Dynamic Source Routing, and like AODV is a passive routing protocol. The DSR helps maintain the routing source where each neighbor node in DSR maintains the entire network path from source to destination [2].

\section{STRUCTURE OF DSDV, AODV AND DSR PROTOCOLS}

DSDV, AODV, and DSR protocols are single-broadcast routing protocols, DSDV is singlebroadcast table based protocol and DSDVand AODV are single-broadcast hybrid based protocols. In figure 3 the structure of the DSDV protocol is shown [2].

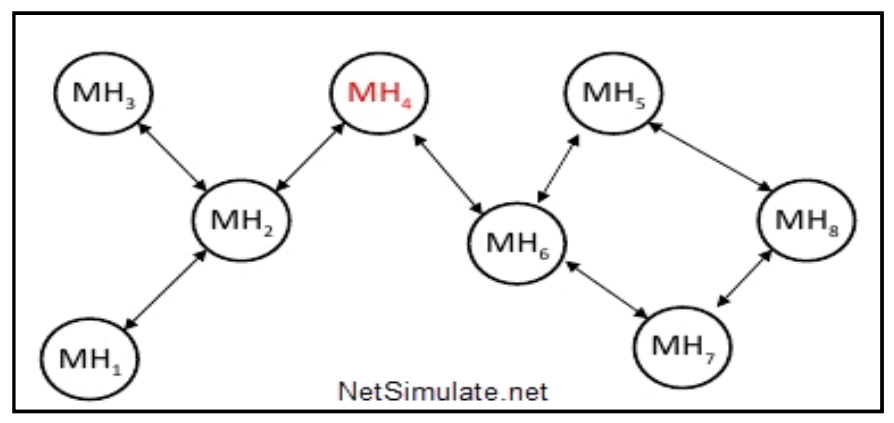

Figure 3.DSDV protocol structure[12]

The DSDV routing protocol is a convenient protocol for ad hoc networks. This extended protocol is the classic distance vector algorithm or DBF. Work has been done in the DSDV routing protocol in order to avoid the loop problem that exists in the DBF algorithm. By combining the input of each routing table with a sequential number to set up routing information, loop formation can be prevented. In the DSDV routing protocol, the routing operations between the nodes are performed using routing tables stored on each node in the ad hoc network. The routing tables in each node specify all the destinations that are available and also the number of distance steps to them. The routing table entry is associated with a sequential number that is created by the destination node. In order to maintain the correct routing table information, the DSDV routing protocol uses both period update and activated path updates. The activated path update is activated with a period update to allow routing information to be disseminated at the earliest possible speed when no change is made in the topology. Updating packages include the available destinations per node, the number of jumps required per destination and the sequence number associated with that route. The data is maintained during the time interval between the first reception and receipt of the best route for each specific destination. The data structure may be configured to delay the announcement of paths that are likely to change, to reduce the fluctuations of the routing tables, and to reduce the number of duplicate paths entered with a sequential number [2] .

The AODV protocol is a demand-driven routing protocol used in ad hoc networks to route. In Figure 4, the structure of the AODV protocol is shown. 


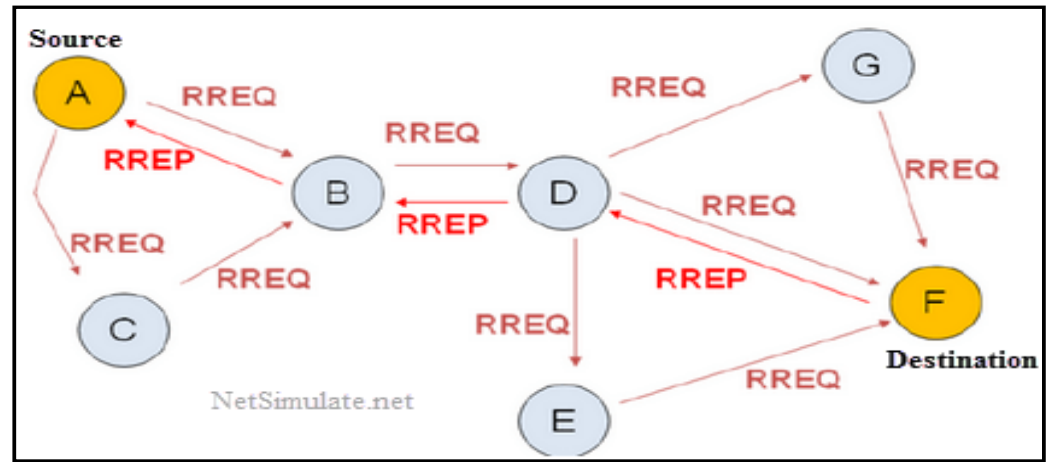

Figure 4: AODV protocol structure[12]

The AODV protocol allows mobile computers, or nodes, to transmit messages among their neighbors, to communicate directly with each other, and as with all reactive need protocols only when a path is required The tracking operation takes place, and of course, when the route is found, it stays stored until it is needed, and then it is no longer kept, and should be re-discovered for the next time. The AODV routing protocol assures that paths do not include the loop and try to find the shortest possible path. Also, the AODV protocol is capable of manually changing the paths, and if there is an error, it could find and replace new paths. The AODV protocol tries to synchronize rapidly with dynamic line conditions, as well as the low volume of processing and memory required are the properties of the AODV algorithm. The AODV protocol uses a destination sequence number to ensure that no loops are created, and also solves the problem encountered in the classical vector-space protocol for counting to infinity. In the AODV protocol, each Node has its own unique sequence number, which is added uniformly. This number is added when the corresponding node notices a change in the network topology. The AODV routing protocol has another significant functionality, and it is the usability of this protocol in all three types of single-broadcasting, multi-broadcasting, and dissemination [2].

The DSR routing protocol (dynamic source routing) also belongs to the class of request-based protocols and is used in ad hoc networks. The DSR protocol allows the node to dynamically identify a path across multiple network jumps through which it reaches its destination, and the route to some destination points is only determined when someone Request to send information to the specified route and destination. In the routing of each packet, there is a complete list of nodes to pass through.

The DSR routing protocol does not use any periodic routing messages, and thus reduces overhead of network bandwidth, maintains battery power, and prevents multiple routing updaters across the network. Instead, the DSR routing protocol is based on support done by MAC layer. Each packet should carry the full address (each jump in the path) from the source to the destination, which is not efficient on large networks. This is due to the fact that the amount of overhead and its corresponding consumption of bandwidth increase with increasing diameter of the network. The DSR protocol does the two main operations of route detection and route maintenance. In Figure 5 , the structure of the above protocol is shown. 


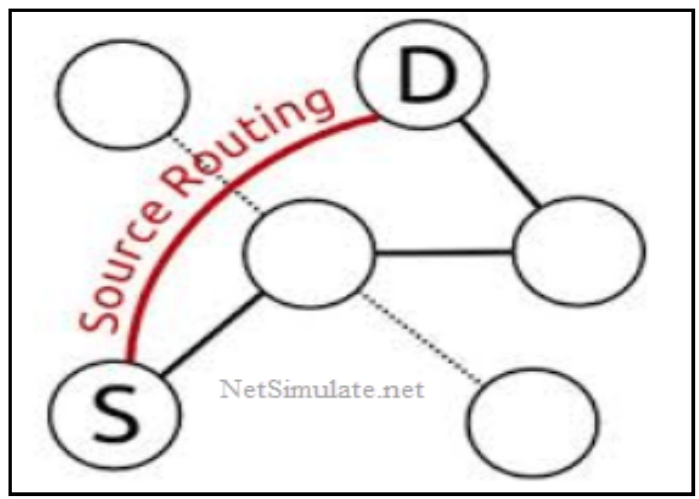

Figure 5: DSR protocol structure [12]

\section{Challenges To DSDV, DSR AND AODV PROTOCOLS}

The DSDV protocol is a table-based routing protocol for mobile networks, in which the route is maintained through routing table exchanges, events, and a timetable. This protocol increases the speed of integration and leads to increased current costs. The above protocol works best for low mobility environments. Examples of problems with the DSDV protocol are the recognition of the best value for parameters such as the maximum seating time for a specific, very difficult and complex destination, which causes the path fluctuations and false alarms, causing the bandwidth will be wasted. The DSDV routing protocol, along with the activated upgrades, also use periodic updates, which results in wasting bandwidth and increased communication costs. Each node of DSDV routing protocol must wait for the next path update driven from destination, and then can update its routing table entries for the respective destination. Another problem is that the DSDV routing protocol does not support multi-paths routing and multicasting. The DSDV protocol also faces the challenge of wasting bandwidth more seriously.

The DSR protocol is designed for a temporary multi-paths wireless network. Unlike other temporary network protocols, the DSR protocol does not require any periodic routing messages on the network. And it does not rely on routing information of the intermediate node. In this protocol, the length of the route affects current routing costs, and broken links in the network do not require local repair in the path maintenance process, which is the main weak point of the protocol, which is unsuitable for a large network with high mobility [4].

The AODV protocol combines some of the capabilities of DSDV and DSR protocolsand when a node needs sending data packets, it creates a path. This protocol uses tables to store routing information, but does not maintain route information for all nodes that are not communicating on the network. The AODV protocol is safer than the other two protocols. One of the major challenges of this protocol is the delays in the establishment of routes, lack of support for the detection and maintenance of multi-paths between each source and destination pair [4].

\section{COMPARISON OF DSDV, DSR AND AODV PROTOCOLS}

DSDV, DSR, and AODV protocols are compared in terms of packet delivery rates, average latency in upload speed, overhead, operational capacity, security, and scalability. In general, the efficiency of these protocols depends on the number, speed of nodes and data traffic. When the 
motion and the number of nodes increase, these parameters change. In Table 1 we show the comparison of these parameters for the above protocols [2].

Table 1: Comparison of DSDV, DSR and AODV protocols [12]

\begin{tabular}{|l|l|l|l|l|l|l|}
\hline $\begin{array}{l}\text { protocol's } \\
\text { name }\end{array}$ & $\begin{array}{l}\text { Package } \\
\text { Delivery } \\
\text { Rate } \\
\text { Criterion }\end{array}$ & $\begin{array}{l}\text { Average } \\
\text { delay }\end{array}$ & overhead & security & Scalability & $\begin{array}{l}\text { Operating } \\
\text { Capacity }\end{array}$ \\
\hline DSDV & High & average & $\begin{array}{l}\text { Depending } \\
\text { on the } \\
\text { amount of } \\
\text { data traffic }\end{array}$ & average & average & average \\
\hline DSR & Average & Low & low & $\begin{array}{l}\text { Depending } \\
\text { on the size } \\
\text { of the } \\
\text { network }\end{array}$ & average & high \\
\hline AODV & High & high & average & high & high & average \\
\hline
\end{tabular}

\section{INTRODUCING SOLUTIONS FOR THE THREE DSDV, DSR AND AODV PROTOCOLS}

TareqEmad Aliet al. [2016] reviewed and compared the performance of the VANET (car case networks), AODV, DSR, OLSR, DYMO, DSDV, ZRP protocols. In this work, a quick review and comprehensive study of the case routing protocols used in VANET are presented. The research domain in the automotive case networks (VANET) is developing very fast. A large number of applications in this process have been adapted for different situations (large roads and cities). Many protocols are presented for different topologies and scenarios, and these protocols face many challenges. VANETs provide links between vehicles that run on the road.Routing protocols in VANET are affected by the car at the highest speed, which makes the connection between most of the vehicles in communication, therefore, the case routing protocols with VANET features for delivering data between vehicles in short time, are matched. The main goal of VANET is to collect a data system between vehicles that run on the road. This process enables cars to communicate with each other for the safety of people on the road. In this work, an attempt is made to compare 6 perceived protocols including AODV, DSR, OLSR, DSDV, ZRP, and DSDV. Components used for comparison in VANET are included packet ratio, end-to-end latency, output, routing algorithm load, received packets, routing packets, packet loss rates, lost packets, jitter average.[5]

RamakantChandrakar et al. [2015] assessed the AODV, DSR and DSDV routing protocols to improve data transfer in mobile case networks. Mobile Case Networks (MANETs) are considered to be very important components for wireless communications because of the growing popularity of mobile devices. The key challenge for future wireless networks is the quality of service for customer satisfaction. The tool that will assist us in the immediate transmission of video media, including video conferencing and webinaring, will be evaluated. Multiple network steps are needed to deliver and share data on the network. Each mobile node acts as a router, which sends data packets to the nearest step, and eventually the packets are delivered to the destination. First, a unique process of mobile case networks and their protocols is described, and then a mobile case network (MANET) consisting of a set of mobile wireless nodes and a fixed wireless server is 
designed using ns-2. In this research work, the simulation of three MANET routing protocols, called AODV, DSR and DSDV, is based on three functional parameters. These parameters include the packet delivery rate (PDR), end-to-end delay, and operational capability.[6]

AkshaiAggarwalet al. [2011] analyzed the performance of AODV, DSDV and DSR in MANETs. Mobile Case Networks (MANETs) are emerging as a new phenomenon of non-infrastructure mobile wireless communication systems. MANETs are widely studied and have technologies that attract a large number of applications. Routing in MANETs is a challenging task due to unpredictable changes in the network topology, which is the result of sequential and random movement of the nodes and the lack of a local control. In this work, we investigate and evaluate the performance of reactive routing protocols, including distance vector based on case demand (AODV), dynamic source routing (DSR), and abstract space vector reactive routing (DSDV). The overall purpose of this work is to analyze the performance of MANET routing protocols in cases of high mobility and low, moderate, and high density congestion scenarios. Unlike military applications, many other MANET applications require a moderate to large movement. Hence, for surveys, the study of the effect of high-mobility motion on the performance of these routing protocols is very important. Performance is analyzed based on the late end-to-end latency, normalized routing load (NRL), packet delivery fraction (PDF), and operational power. Simulation results show that AODV offers better performance than DSR and DSDV.[7]

NityaThageleet al. [2018] critically examined the AODV, DSDV, and DSR protocols in the presence of malicious nodes in mobile case networks. Mobile Case Networks (MANETs) are decentralized and automated wireless systems. A mobile case network is a process for collecting wireless networks and building an independent network without any infrastructure. Routing is a fundamental and significant issue and a major challenge in case networks. Many of the routing protocols are presented to improve routing functionality and reliability. The research work outlines a summary of the characteristics of routing protocols based on different performance metrics such as packet delivery fraction, average delay, normalized routing load, and operational power in low-mobility mode and low traffic networks as well as high-mobility mode and hightraffic network with presence of malicious nodes.[8]

Brijendra Kumar Joshiet al. [2016] evaluated the security of the AODV protocol under the DOS and Wormhole attacks. A MANET is defined as a set of mobile wireless nodes that share a common wireless channel without any central unit. In recent years, many routing approaches have been introduced to use MANET in states, business applications, and military applications. MANETs have some qualities like dynamic nature, decentralized support, and non-infrastructure procedures that are prone to attacks. Security is a major issue in the design of routing protocols in MANETs. In this paper, we present a safety analysis of the routing protocol in the distance vector based on the case and general demand, especially under different types of attacks.[9]

PramodMutalik et al. [2016] provided a comprehensive review of the AODV, DSR and DSDV routing protocols for Intelligent Transportation Systems (ITS) in metro cities to secure road traffic using VANET Traffic Analysis. Road traffic safety can be improved and have a better ability to complete inter-car communications by implementing new cellular technologies such as VANETs. VANETs, based on combined network architecture, are application scenarios of switching positions that do not include the location and change of a node. The principle behind the design of the routing protocol lies in the fundamental differences between VANETs. The Intelligent Transportation System (ITS) serves as a solution to the above issues. AODV, 
DSDVand Dynamic Source Routing (DSR) have been studied in this work. Routing algorithms are presented to improve accuracy based on urban environmental features such as data loss, data collisions, vehicle congestion, and end-to-end delay.[10]

SaimaZafar et al. [2016] analyzed latency and operational capabilities of AODV, DSDV, and DSR routing protocols in mobile case networks. Mobile case networks are presented in order to play a vital role in inclusive networks for supporting their mobility without relying on infrastructure-based design. Conversely, the same feature makes routing in these networks more challenging than conventional wired networks. As a result, routing protocols that are designed for wired networks are not suitable for these networks. A number of routing protocols have emerged over the past few years that can generally classify proactive and reactive routing protocols. In this work, we evaluate and compare three important routing protocols from two sets of power components, end-to-end latency and packet delivery fractions. This comparison is critical to understanding the requirements and challenges of routing protocols in mobile case settings and structuring shapes for designing new routing protocols in the future. The simulation results based on the simulations presented in the network simulator (NS2) showed that the Dynamic Source Routing Protocol (DSR) had the best performance compared to the AODV and DSDV when the size of the network was large and the nodes' movement was high.[11]

\section{Conclusion}

In this research, DSDV, DSR and AODV protocols were evaluated in VANET and MANET networks. In fact, the efficiency of these protocols depends on the number and velocity of the nodes and the traffic load of the data. When the movement and population of the nodes increase, the routing overhead increases and prevents the delivery of data packets.

The AODV protocol uses digital signing in its routing messages to ensure integrity and credibility.

This is one of the things that makes the protocol safer than the other two protocols.

The DSR protocol in mobile case networks is more appropriate in terms of end-to-end latency of the packet delivery fraction throughput, from the other two protocols in large networks with large moving nodes.

MANET networks that have civilian applications have large amounts of movement. In the reviews, the depreciation of the delivery packet of the normalized routing burden throughput of the protocol AODV performs better.

One of the major challenges faced by the DSDV protocol is bandwidth loss because this protocol uses periodic updates along with the active update. Or, in order to determine the best sitting time for a particular destination it causes fluctuations in the number of false alarms. 
International Journal on Ad Hoc Networking Systems (IJANS) Vol. 8, No. 3, July 2018

\section{REFERENCES}

1. Mohammad Ali Nazeri, MoeineSarvy, MortezaRomuzi, HamidehBabaei, "Comparison and Evaluation of DSDV, DSR and AODV Routing Protocols in Car Ad hoc Network", Second National Computer Conference, Information Technology of Iran, 2016.

2. Mohammad Ghoddini, "Comparison of the Efficiency of single-broadcast and multi-broadcast Routing Protocols in a Car Network", Second Iranian National Congress on New Technologies, Tehran, 2011.

3. Venkatesh, A Indra, R Murali Routing Protocol s for Vehicular Adhoc Networks (VANETs): A Review Journal of Emerging Trends in Computing and Information Sciences ISSN 2079-8407 Vol. 5, No. 1 January 2014

4. FahadTahaAL -Dhief, NaseerSabri, M.S. Salim, S. Fouad, S. A. Aljunid," MANET Routing Protocols Evaluation: AODV, DSR and DSDV Perspective", MATEC Web of Conferences 150, 060242018.

5. TareqEmad Ali, Layth A. Khalil al dulaimi, Yamaan E. Majeed," Review and Performance Comparison of VANET Protocols: AODV, DSR, OLSR, DYMO, DSDV \& ZRP”, 2016 Al-Sadeq International Conference on Multidisciplinary in IT and Communication Science and Applications (AICMITCSA)- IRAQ, IEEE, 2016.

6. RamakantChandrakar, Ajay Kushwaha, NehaChoubey,"Evaluation of Routing Protocols AODV,DSR and DSDV to enhance videotransmission over Mobile Ad Hoc Networks," SPACES2015, Dept of ECE, K L UNIVERSITY.

7. AkshaiAggarwal, Savita Gandhi, NirbhayChaubey," PERFORMANCE ANALYSIS OF AODV, DSDV AND DSR IN MANETS", International Journal of Distributed and Parallel Systems (IJDPS) Vol.2, No.6, November 2011, IEEE, 2011.

8. NityaThagele, PriyankaTripathi, "A Critical Review of AODV, DSDV and DSRProtocol Presence of Malicious Node in Mobile Ad-Hoc Network", 2018.

9. Brijendra Kumar Joshi, MeghaSoni," Security Assessment of AODV Protocol under Wormhole and DOS Attacks", IEEE, 2016.

10. PramodMutalik, Nagaraj S, Vedavyas J," A Comparative study on AODV, DSR and DSDV routing protocols for Intelligent Transportation System (ITS) in Metro cities for Road Traffic Safety using VANET Route Traffic Analysis (VRTA)", 2016 IEEE International Conference on Advances in Electronics, Communication and Computer Technology (ICAECCT) RajarshiShahu College of Engineering, Pune India, IEEE, 2016.

11. SaimaZafar, Hina Tariq, KanzaManzoor," Throughput and Delay Analysis of AODV, DSDV and DSR Routing Protocols in Mobile Ad Hoc Networks", International Journal of Computer Networks and Applications (IJCNA) Volume 3, Issue 2, 2016.

12. https://www.noavarangermi.ir/ 